\title{
Zonificación del riesgo de tsunami en el centro-sur de Chile ${ }^{1}$
}

\author{
Marcelo Lagos ${ }^{2}$
}

\begin{abstract}
RESUMEN
Integrando modelación numérica de tsunamis, técnicas geomáticas y nuevos criterios desarrollados por la investigación del riesgo de tsunami, se desarrolla una metodología para zonificar riesgo y se aplica en un área costera del centro-sur de Chile. El método utiliza factores que inciden en el riesgo de tsunami, como parámetros hidrodinámicos de la amenaza y aspectos físicos y socioeconómicos de la vulnerabilidad. Los factores de riesgo son integrados mediante técnicas de Evaluación Multicriterio y Sistemas de Información Geográfica. Los resultados de la zonificación, evidencian cómo las características del lugar, su localización, el comportamiento diferenciado de la amenaza, y los mayores porcentajes de pobreza y su concentración, condicionan los niveles de riesgo. Esta información se transforma en la base para futuros estudios de planificación territorial, que permitan disminuir el riesgo de desastre por tsunami.
\end{abstract}

Palabras clave: Tsunami, modelación de tsunami, riesgo, evaluación multicriterio.

\begin{abstract}
Integrating tsunami numerical modeling, geomatics techniques and new criteria developed by the tsunami risk research, develops a methodology for risk zoning and applied in a coastal area in south-central Chile. The method uses factors that influence the tsunami risk, as hydrodynamic parameters of the threat and physical and socioeconomic aspects of vulnerability. Risk factors are integrated through multicriteria evaluation techniques and Geographic Information Systems. The zoning results show that the local characteristics and their location, together with the concentration of poverty levels, establish spatial differentiated risk levels. This information builds the basis for future applied studies in land use planning, which would reduce the risk of tsunami disaster.
\end{abstract}

Key words: Tsunami, tsunami modeling, risk, multicriteria evaluation.

\footnotetext{
1 Investigación financiada por el Proyecto FONDECYT 11090210. Artículo recibido el 28 de junio de 2012, aceptado el 30 de julio de 2012 y corregido el 9 de octubre de 2012.
}

\footnotetext{
2 Laboratorio de Investigación de Tsunami, Instituto de Geografía, Pontificia Universidad Católica de Chile. (Chile). E-mail: mlagoslo@uc.cl
} 
Chile es uno de los principales generadores de tsunami en el océano Pacífico. Su historia registra decenas de tsunamis destructivos y su prehistoria confirma su recurrencia (Lagos y Cisternas, 2008). Sin embargo, si bien los tsunamis son una amenaza permanente para toda comunidad costera localizada frente a una zona de subducción, lamentablemente, su peligrosidad sigue siendo subestimada. En Chile, ejemplos recientes son la magnitud e impacto que tuvo el tsunami de campo cercano de 2010 (Lagos et al., 2010; Fritz et al., 2011); y los daños diferenciados que generó el tsunami de campo lejano originado el 2011 en las costas de Tohoku, Japón (Lagos \& Haro, 2011).

Ciertamente, en Chile resulta muy fácil olvidar. Independiente de tener un pasado de territorios costeros que recurrentemente han sido inundados y destruidos por tsunamis, es común observar a escasos metros de la costa y bajo la altitud de $10 \mathrm{~m}$, viviendas, escuelas, asilos de ancianos, municipalidades e infraestructura crítica de diversa jerarquía (Lagos et al., 2008). Todo lo anterior evidencia lo poco que se aprendió después del gran tsunami chileno de 1960 (DNHA, 1961) y confirma la escasa consideración del peligro de tsunami en la localización de asentamientos humanos costeros y su planificación territorial (Andrade et al., 2010).

Como medida reactiva, después de los daños generados por el tsunami chileno de febrero de 2010, se ha dado relevancia a nuevos estudios de peligro y riesgo de tsuna$m i$, esfuerzos inéditos que buscan apoyar el proceso de reconstrucción de las localidades costeras afectadas y la formulación y/o actualización de los respectivos Instrumentos de Planificación Territorial (UC, 2010a; UC, 2010b; UBB, 2010). Si bien todo nuevo estudio es bienvenido, la urgente necesidad de comprender el riesgo de tsunami, no es solo de los asentamientos que fueron inundados en el tsunami de 2010, sino que es un problema de toda la costa de Chile.

La demanda de estudios de riesgo de tsunami, necesariamente implica el desarrollo y aplicación de metodologías robustas que permitan discriminarlo. Actualmente, la optimización de métodos para conocer y espacializar el potencial comportamiento de tsunamis en áreas costeras se encuentra en continuo desarrollo. Mejoras en las técnicas de modelación numérica (Satake et al., 2007; Sinolakis \& Kanoglu, 2009; Arcas et al., 2010; Yamazaki et al., 2010) y en el diseño de mapas de peligro de tsunami (Sato et al., 2003; Dengler et al., 2003; Sugimoto et al., 2003; González et al., 2005; Imamura, 2009; Lagos et al., 2011), son evidencia de un permanente interés por integrar el estudio de estos fenómenos en la comprensión del riesgo.

El conocimiento del riesgo de tsunami es el punto de partida para generar comunidades resilientes ante la amenaza de tsunami (Bernard, 2001). Su comprensión se sustenta en la elaboración de zonificaciones de riesgo, que buscan explicar el porqué del riesgo, espacializando sus variaciones en un territorio dado, mediante la integración de un conjunto de elementos que caracterizan la amenaza y la vulnerabilidad de los asentamientos expuestos (IOC, 2009).

Ciertamente, en localidades costeras donde ha ocurrido un tsunami reciente (ej. tsunami chileno de 2010), la caracterización del comportamiento real de la inundación y el impacto territorial producido por el tsunami, facilita la comprensión de la peligrosidad y de las condiciones multifactoriales de la vulnerabilidad. Esta información es un input valioso para las futuras zonificaciones de riesgo, sobre todo para las numerosas localidades que han olvidado su pasado de tsunamis, simplemente porque no ha ocurrido uno recientemente.

En este escenario, se propone un método para zonificar el riesgo de tsunami y se aplica en una localidad del centro-sur de Chile, que posee un pasado de inundaciones de tsunami, como gran parte de las localidades costeras de Chile. La zonificación busca comprender el proceso diferenciado de inundaciones generadas por tsunami y su relación con la vulnerabilidad, de modo de responder preguntas que permitan optimizar la discriminación del riesgo.

\section{Descripción del área de estudio}

La zonificación de riesgo de tsunami se realiza en el curso inferior del estuario del río 
Maullín, Región de Los Lagos, Chile. Localizado entre los $41^{\circ} 34^{\prime}$ y $41^{\circ} 39^{\prime}$ de latitud Sur, y $\operatorname{los} 73^{\circ} 43^{\prime}$ y $73^{\circ} 34^{\prime}$ de longitud Oeste (ver Figura $\mathrm{N}^{\circ} 1$ ). En un contexto rural, el área de estudio presenta asentamientos humanos concentrados y dispersos, destacando la localidad de Maullín como el asentamiento principal. Las partes bajas del estuario fueron ampliamente afectadas por el tsunami chileno de 1960. Las máximas olas observadas alcanzaron en la costa alturas cercanas a los 10 metros. El tsunami dejó un saldo total de 122 muertes, 115 corresponden al pueblo de Quenuir, que fue arrasado completamente; 15 fallecieron en el sector de San Pedro Nolasco; y dos en el poblado de Maullín (Atwater et al., 1999).

Más de cinco décadas han transcurrido desde el tsunami de 1960 en Maullín, tiempo suficiente para olvidar. Hecho confirmado por la localización de nuevas viviendas e infraestructura crítica muy cercanas al mar, incrementando el riesgo de desastre (Lagos et al., 2008). Sin embargo, consecuencia del tsunami chileno de 2010, la percepción del riesgo ha cambiado, transformándose en una preocupación para la comunidad en general. Hoy, como medidas de mitigación, Maullín ha realizado simulacros de evacuación e instalado señaléticas de peligro de tsunami. No obstante, sin un claro conocimiento de cómo se comportará una potencial inundación y qué áreas se encuentran en riesgo, es difícil ordenar territorios costeros y desarrollar planes de respuesta efectivos ante la amenaza de tsunami.

\section{Materiales y métodos}

\section{Modelación de tsunamis y selección de escenarios}

Para simular el escenario de peligro de tsunami, se utilizó el código TUNAMI-N2 (Goto et al., 1997), modelo numérico ampliamente utilizado y validado con datos de recientes tsunamis (Dao \& Tkalich, 2007; Sugawara \& Goto, 2012). Para la modelación, se generó un dominio de integración compuesto de datos topográficos y batimétricos del centro-sur de Chile, con una longitud aproximada de mil kilómetros desde Concepción por el norte $\left(36^{\circ} 58^{\prime} \mathrm{S}\right)$, hasta la península de Taitao por el sur $\left(46^{\circ} \mathrm{S}\right)$, y un ancho aproximado de $400 \mathrm{~km}$ desde el océano Pacífico $\left(76^{\circ} 30^{\prime} \mathrm{W}\right)$ hasta el interior

Figura $\mathrm{N}^{\circ} 1$

Localización del área de estudio
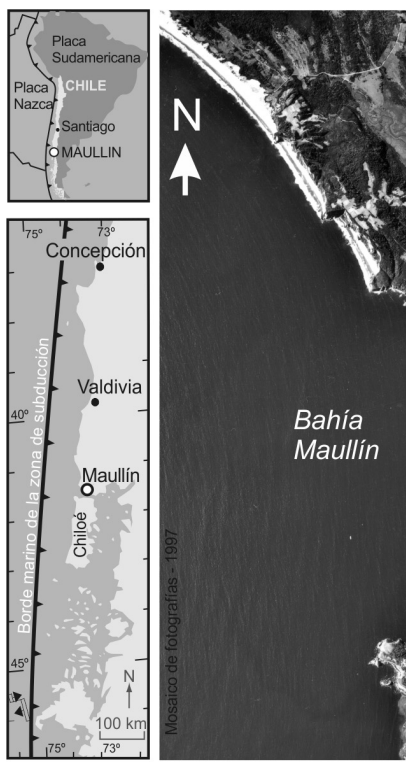

Fuente: Elaboración propia. 
del continente $\left(72^{\circ} 15^{\prime} \mathrm{W}\right)$. Las fuentes de información utilizadas incluyen el levantamiento The General Bathymetric Chart of the Oceans (GEBCO), Shuttle Radar Topography Mission (SRTM), cartas náuticas del Servicio Hidrográfico y Oceanográfico de la Armada de Chile (SHOA) y levantamientos detallados en terreno. La resolución espacial de celda para modelar inundación fue de 3" ( 90 m) y el tiempo de simulación cuatro horas.

De acuerdo a Lagos \& Cisternas (2008), se seleccionaron dos escenarios capaces de generar tsunamis en Maullín, un evento probable que sería un tsunami que podría manifestarse en el corto o mediano plazo, y un peor escenario, que sería un tsunami de gran impacto. Ambos eventos se pueden caracterizar considerando para el primer escenario el tsunami de 1837 y para el segundo el gran tsunami de 1960.

El Cuadro $N^{\circ} 1$ presenta los parámetros macrosímicos utilizados para generar las condiciones iniciales de los tsunamis de 1837 y 1960. Para el caso del tsunami de 1960, se evaluaron previamente once combinaciones de parámetros macrosísmicos propuestos por Kanamori \& Cipar (1974), Cifuentes (1989) y Barrientos \& Ward (1990). Seleccionándose los propuestos por Barrientos \& Ward (1990), debido al mejor ajuste de los resultados de la modelación, con datos observados de alturas del tsunami (Sievers et al., 1963) y depósitos de tsunami de 1960 en Maullín (Lagos y Cisternas, 2004; Cisternas et al., 2005).

\section{Zonificación del riesgo de tsunami}

La zonificación de riesgo de tsunami se desarrolló mediante la integración en Sistemas de Información Geográfica (SIG) de planos de información como criterios que componen y condicionan el proceso de riesgo. Para ello, se utilizó el método de Evaluación Multicriterio (EMC). La EMC puede definirse como un conjunto de técnicas y modelos orientados a asistir procesos de decisión a problemas espaciales complejos, permitiendo generar alternativas en base a múltiples criterios (Chen et al., 2001). En este caso, los criterios corresponden a los factores de amenaza y vulnerabilidad.

Basado en los daños producidos por tsunami y parámetros recomendados para el diseño de mapas de peligro (Bernard \& Robinson, 2009), se seleccionaron como criterios de amenaza de tsunami en el área de inundación, la magnitud de la profundidad de inundación y la velocidad de la corriente. En tanto, los criterios para identificar vulnerabilidad, se concentraron en factores físicos y socioeconómicos, de acuerdo a las recomendaciones de UN-ISDR (2004) y las características del área de estudio, siendo seleccionados la localización en zona costera, distancia

Cuadro $\mathrm{N}^{\circ} 1$

Parámetros de falla utilizados para simular tsunamis

\begin{tabular}{|c|c|c|c|c|c|c|c|c|c|c|}
\hline 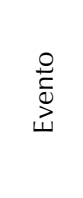 & 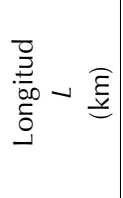 & 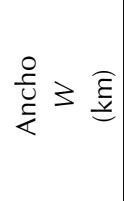 & 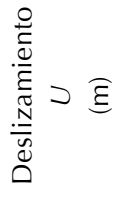 & 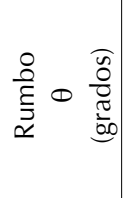 & 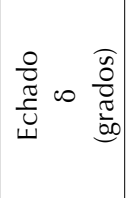 & 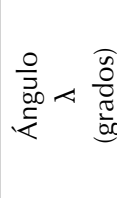 & 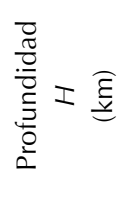 & 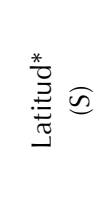 & 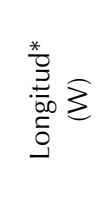 & $\Sigma^{0} \overline{\underline{E}}$ \\
\hline 1837 & 390 & 180 & 11 & 355 & 18 & 90 & 15 & 45.324 & 75.723 & $2.3 \times 10^{22}$ \\
\hline 1960 & 850 & 130 & 17 & 7 & 20 & 105 & 4 & 45.324 & 75.723 & $0.9 \times 10^{23}$ \\
\hline
\end{tabular}

* Coordenada de esquina SW.

Fuente: Elaboración propia en base a Lagos y Cisternas (2008) y Barrientos \& Ward (1990). 
a sitio seguro, pendiente del terreno, altitud, pobreza y densidad de habitantes.

Los criterios de amenaza se obtuvieron de la modelación numérica del tsunami de 1960, el peor escenario. Para ello, se calculó, a intervalos de cinco minutos, el comportamiento espacio-temporal máximo de cada parámetro hidrodinámico, sobre la superficie inundada y por un total de cuatro horas. La profundidad de inundación se expreso en metros sobre el terreno y la velocidad de la corriente en metros por segundo.

Para los criterios de vulnerabilidad, la localización en zona costera se clasificó en cuatro niveles: zona de seguridad; área bajo la zona de seguridad; localizarse en el área de inundación del peor escenario; y localizarse en el área de inundación del evento probable y peor escenario. La zona de seguridad se definió a partir de la altitud alcanzada por el runup máximo del peor escenario, más el rango mareal de sicigia en Maullín. Este criterio, propuesto por Venturato et al. (2004), permite estar preparado en el caso que un tsunami se manifieste en las costas durante la marea más alta registrada en el lugar, situación que aumentaría los alcances de la inundación producida por un tsunami.

La distancia a zona de seguridad se calculó sobre la superficie rugosa de un modelo digital de elevación (MDE), elaborado con curvas de nivel con equidistancia de un metro. En base al mismo MDE, se calculó la pendiente del terreno en grados y la altitud en metros sobre el nivel medio del mar.

La densidad de habitantes en el área de estudio, se determinó a través del total de población del Censo de Población y Vivienda del año 2002 (INE, 2003). Para el área urbana se digitalizaron todas las manzanas urbanas del distrito de Maullín. Para el caso del área rural, se digitalizó, de manera puntual, cada vivienda graficada en los planos censales de los sectores rurales. La vinculación con información de población se realizó en el programa REDATAM - SP.

Por último, el criterio pobreza se generó mediante el Método Integrado de Medición de la Pobreza (MIP) propuesto por el PNUD (1990) e ILPES (1995), que considera la combinación de los métodos de Necesidades Básicas Insatisfechas (NBI) relativo a las carencias físicas de las viviendas y el método de Línea de Pobreza (LP) que mide indirectamente el ingreso en función de la vulnerabilidad social del jefe de hogar. Los datos se obtuvieron del Censo 2002 (INE, 2003).

Una vez generadas las bases de datos de cada criterio, estos fueron remuestreados y homologados a una matriz común, compuesta de 137 columnas y 100 líneas que cubre el área de estudio, con celdas de 100 metros de resolución espacial. Luego, cada criterio fue ponderado y normalizado para ser integrado en la Evaluación Multicriterio. Para definir la importancia de cada criterio, se realizaron funciones mediante el método de lógica borrosa (Ghribi, 2005). Estas funciones permitieron definir en cada criterio espacial, el grado de pertenencia que tiene cada píxel a un determinado conjunto; en este caso, conjunto de datos espaciales que permiten identificar el grado de amenaza o vulnerabilidad ante tsunami. Para cada criterio espacial, el grado de pertenencia fue normalizado en un rango de valores de celda de 0 a 1 . Las funciones definidas son las siguientes:

\section{Funciones utilizadas para ponderar y estandarizar los criterios de amenaza de tsunami}

Para la velocidad de la corriente de tsunami se utilizó una función en forma de J monotónicamente incremental (ver Figura $\mathrm{N}^{\circ} 2$ ). Los valores de velocidad se tomaron de Shuto (1993), Walsh et al. (2005) y Koshimura \& Yanagisawa (2007). Donde el primer punto de control $(1.5 \mathrm{~m} / \mathrm{s})$ representa la velocidad del flujo donde una persona puede tener dificultades para mantenerse en pie. A partir de esta velocidad se incrementa el grado de amenaza rápidamente, siendo el punto de inflexión los $5 \mathrm{~m} / \mathrm{s}$, velocidad donde el peligro es constante. En el caso de viviendas, Koshimura \& Yanagisawa (2007) demuestran que velocidades de la corriente cercanas a $2 \mathrm{~m} / \mathrm{s}$, se traducen en el inicio de daños significativos, transformándose en destructivos cerca de los $5 \mathrm{~m} / \mathrm{s}$.

Respecto a la profundidad de la inundación, también se utilizó una función en forma de J monotónicamente incremental 
(ver Figura $\mathrm{N}^{\circ} 2$ ). De acuerdo a Walsh et al. (2005), se definieron los puntos de control, considerando los rangos de profundidad: 0-0.5 m, 0.5-2 m y mayor a $2 \mathrm{~m}$. Estos rangos de profundidad de inundación representan aproximadamente la altura de las rodillas o menos, sobre las rodillas y más arriba de la cabeza, y mucho más arriba de la cabeza de una persona, representando niveles de riesgo para la seguridad de las personas.

\section{Funciones utilizadas para ponderar y estandarizar los criterios de vulnerabilidad ante tsunami}

La altitud se consideró como un umbral entre la seguridad y el riesgo. Se aplicó una función lineal de forma monotónica decreciente (ver Figura $\mathrm{N}^{\circ} 3$ ). El punto de control (a) corresponde a la altitud a partir de la cual comienza a disminuir la exposición. Esta cota se determinó a partir de la altitud alcanzada por el runup máximo del tsunami de 1960 (peor escenario) en Maullín, más el rango mareal de sicigia en el lugar. El runup máximo en Maullín fue de 7,14 m. En tanto, el rango mareal de sicigia alcanza 2,41 m (SHOA, 2003). La suma de estos valores da $\sim 10 \mathrm{~m}$, por lo tanto, bajo esta altitud, el nivel de exposición es máximo, disminuyendo al mínimo al alcanzar altitudes superiores a los
$30 \mathrm{~m}$ (punto de control (b). Como complemento, se debe destacar que para el tsunami chileno de 2010, en zonas inundadas interiores, la cota $10 \mathrm{~m}$ no fue sobrepasada.

La distancia a sitio seguro se determinó mediante una función lineal de forma monotónica incremental (ver Figura $N^{\circ} 3$ ). El punto de control (a) corresponde a estar localizado en el límite de seguridad (cota $10 \mathrm{~m}$ ), por lo tanto, la distancia es $0 \mathrm{~m}$. A medida que uno se aleja de este límite, aumenta la exposición. El punto de control (b), corresponde a la distancia que una persona caminando puede recorrer antes que arribe el tsunami a la costa. Efectivamente, la guía de preparación en caso de tsunami elaborada por IOC (2008), recomienda considerar velocidades de evacuación caminando, como un parámetro promedio.

Los resultados de la modelación de un tsunami como el de 1960, confirman el comienzo de la inundación a partir de 25 minutos después del terremoto, tiempo que coincide con el testimonio de sobrevivientes. Asumiendo una velocidad de escape promedio caminando de 3,6 km/h (equivalente a 1 $\mathrm{m} / \mathrm{s}$ o $60 \mathrm{~m} / \mathrm{min}$ ), se puede llegar en el tiempo límite de 25 minutos a la zona de seguridad, siempre y cuando el individuo se localice a no más de $1,5 \mathrm{~km}$ de ella $(60 \mathrm{~m} / \mathrm{min} \times 25 \mathrm{~min}$

Figura $\mathrm{N}^{\circ} 2$

Funciones de ponderación y estandarización de criterios de amenaza de tsunami

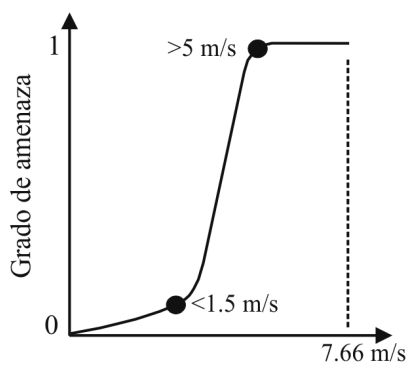

Velocidad de la corriente

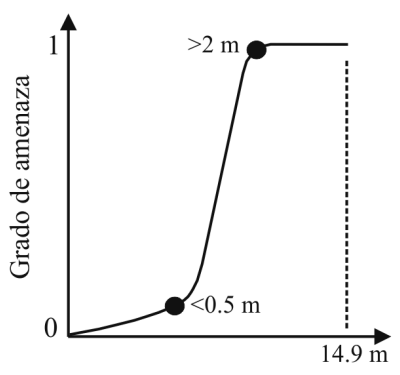

Profundidad de la inundación

*El valor en el eje x representa el máximo valor obtenido en el área de estudio. Fuente: Elaboración propia. 
Figura $\mathrm{N}^{\circ} 3$

Funciones de ponderación y estandarización de criterios de vulnerabilidad por tsunami

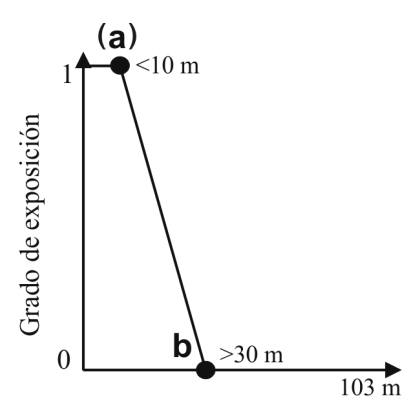

Altitud

(b)

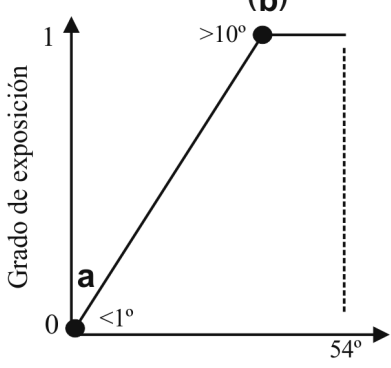

Pendiente del terreno

(b)

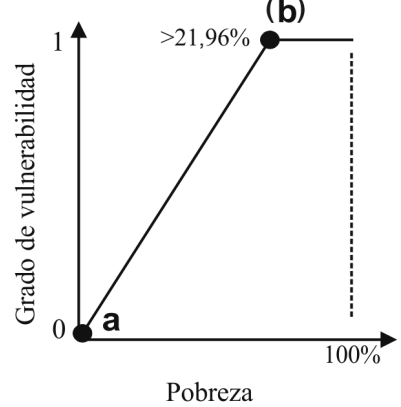

(b)

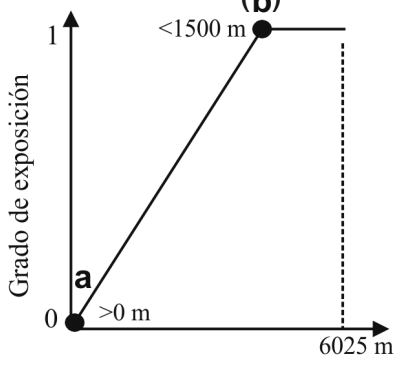

Distancia a sitio seguro

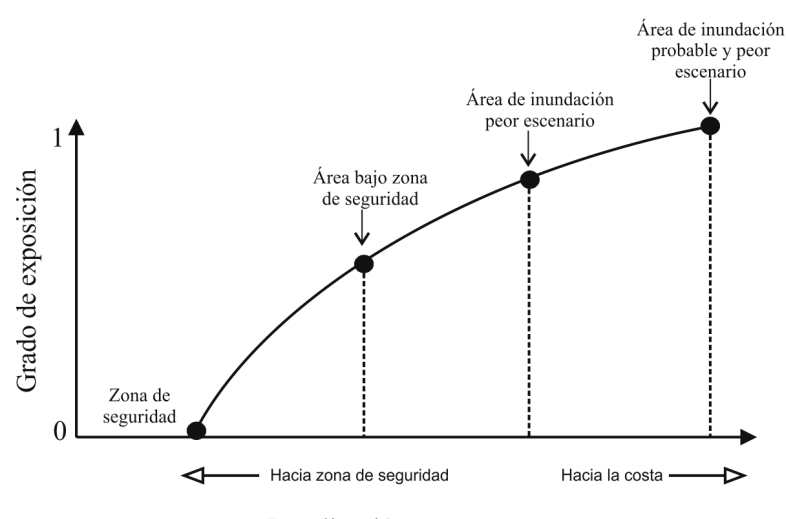

Localización en zona costera

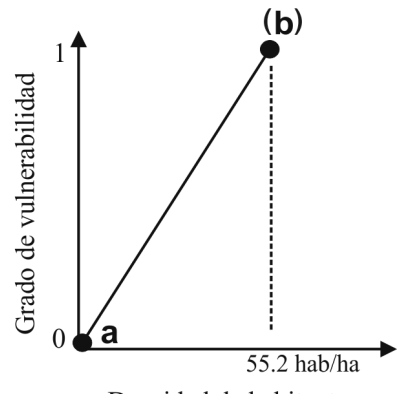

Densidad de habitantes

*El valor en el eje x representa el máximo valor obtenido en el área de estudio (Excepto en los criterios Localización en zona costera y Pobreza).

Fuente: Elaboración propia.

$=1.500 \mathrm{~m})$. Por lo tanto, quienes se localizan a más de $1,5 \mathrm{~km}$ de la zona de seguridad, presentan máxima exposición.

La pendiente del terreno se consideró como un factor que influye en el proceso de escape a zonas de seguridad. El supuesto es que a mayor pendiente se dificulta el escape, en cambio, a menor pendiente el escape se facilita. Para ello, se determinó una función lineal de forma monotónica incremental (ver Figura $N^{\circ} 3$ ). El punto de control (a) corres- 
ponde a una superficie plana menor a $1^{\circ}$ de pendiente. El punto de control (b) indica el grado de pendiente donde notoriamente aumenta el esfuerzo físico para recorrer largas distancias, como las planicies de Maullín. A partir de (b), el grado de exposición se hace constante.

La localización en zona costera se determinó mediante una función convexa monotónicamente incremental (ver Figura $N^{\circ} 3$ ). Donde el menor grado de exposición corresponde a localizarse en la zona de seguridad, y el máximo grado de exposición se aplica cuando la localización coincide con el área de inundación estimada para un tsunami probable y un tsunami excepcional (peor escenario).

Como agente de vulnerabilidad multifactorial, la pobreza se integró mediante una función lineal de forma monotónica incremental (ver Figura $\mathrm{N}^{\circ} 3$ ). El punto de control (a) corresponde al porcentaje de la población no pobre. A medida que se manifiesta pobreza, esta aumenta linealmente el grado de vulnerabilidad. El punto máximo (b) corresponde al porcentaje de pobreza mayor al promedio regional, umbral donde el grado de vulnerabilidad es constante y máximo. Para el caso de la Región de Los Lagos, el porcentaje de pobreza promedio, corresponde a $21,96 \%$ (punto de control b).

Por último, para la densidad de habitantes se aplicó una función lineal de forma monotónica incremental (ver Figura $N^{\circ} 3$ ). Se consideró que a medida que aumenta la densidad de habitantes, aumenta la vulnerabilidad. El punto de control (a) corresponde a una superficie sin habitantes, por lo que su grado de vulnerabilidad es 0 . A medida que aumenta la densidad poblacional, aumenta su grado de vulnerabilidad. El punto máximo (b) corresponde a la mayor densidad registrada en el área de estudio. En el caso de Maullín, por ser un área mayoritariamente rural, la máxima densidad calculada fue de 55,2 hab/ha.

\section{Integración de criterios y definición de capas-objetivo}

Una vez ponderados y normalizados los criterios, mediante EMC se definieron capas- objetivo para espacializar: I. la amenaza de tsunami diferenciada (ATD); II. la exposición (E); y III. la vulnerabilidad socioeconómica (VS). Inputs utilizados para zonificar el riesgo de tsunami.

Para espacializar la amenaza de tsunami diferenciada (ATD), se integró la profundidad de inundación y la velocidad de la corriente. Considerando que a medida que aumenta la magnitud de ambas variables, su potencial de daño se incrementa, se asignaron a cada criterio pesos equivalentes a 0.5. Es decir, ambos criterios poseen la misma importancia. Por lo tanto, en las celdas donde se presentan los mayores parámetros hidrodinámicos, existe mayor peligro de tsunami.

Para determinar la exposición (E) ante tsunami, se integró la localización en zona costera; la distancia a sitio seguro; la pendiente del terreno y la altitud. En este caso, para atribuir pesos diferenciados a los criterios, se utilizó el algoritmo de jerarquías Analíticas AHP. Los pesos asignados a cada criterio fueron: 0.42 para la localización en zona costera y la distancia a sitio seguro; 0.04 a la pendiente del terreno y 0.12 a la altitud. La validez de los pesos asignados se calculó mediante el Índice de Consistencia propuesto por Saaty (1987), arrojando una razón de consistencia aceptable $(\mathrm{IC}=0.08)$.

Para espacializar la vulnerabilidad socioeconómica (VS), se integraron los criterios pobreza y densidad de habitantes. Considerando que en el área de estudio la vulnerabilidad ante tsunami aumenta a mayor pobreza y densidad de habitantes, se asignaron a cada criterio pesos equivalentes a 0.5. Es decir, ambos criterios poseen la misma importancia.

El producto de la exposición (E) y la vulnerabilidad socioeconómica (VS) permitieron obtener la vulnerabilidad (V), la que combinada con la amenaza de tsunami diferenciada (ATD), permitió zonificar el riesgo de tsunami (ZRT). Los resultados se espacializaron en mapas, definiéndose cinco rangos mediante el método de los quiebres naturales. Los rangos se dividieron en muy alto, alto, medio, bajo y muy bajo. La Figura $\mathrm{N}^{\circ} 4$ muestra el esquema metodológico resultante. 
Figura $\mathrm{N}^{\circ} 4$

Inputs utilizados para zonificar el riesgo de tsunami

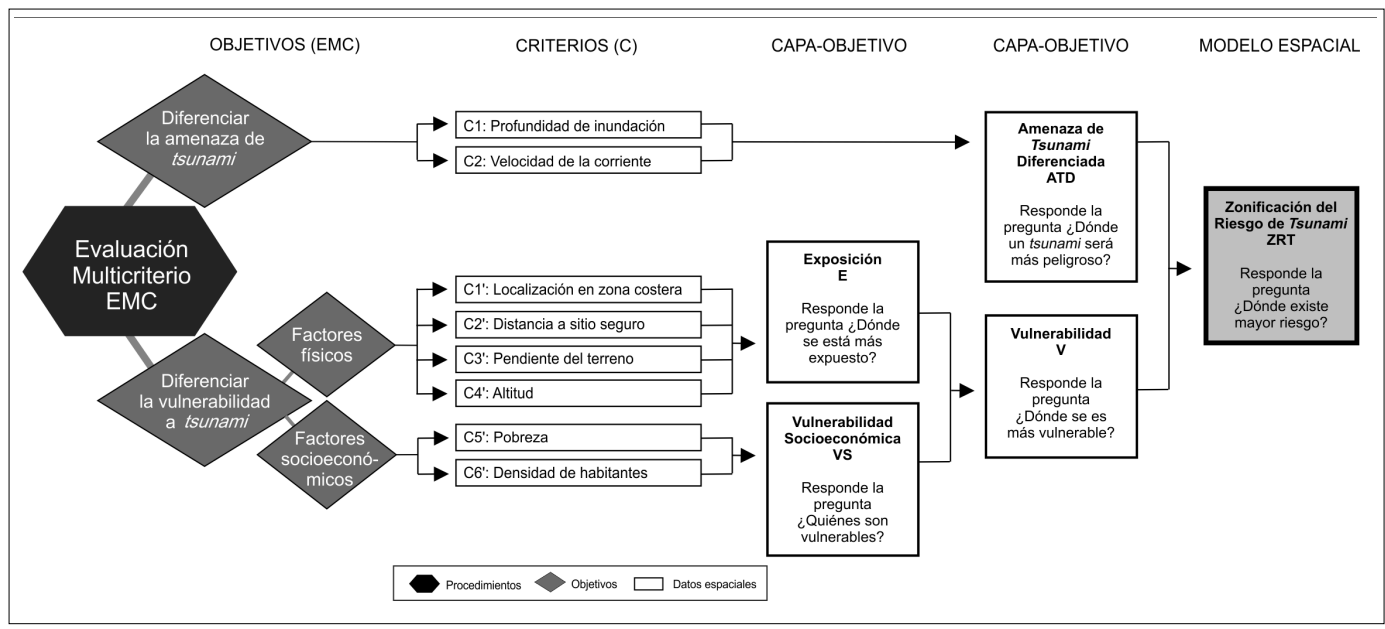

Fuente: Elaboración propia.

\section{Zonificación de riesgo de tsunami en el curso inferior del estuario del río Maullín}

Todos los criterios se transformaron en matrices georreferenciadas. La Figura $N^{\circ} 5$ presenta la espacialización de los factores de amenaza y vulnerabilidad en el curso inferior del río Maullín. La combinación de ellos, fue la base para generar los inputs de las capasobjetivo (Ver Figura $N^{\circ} 6$ ).

La integración de las máximas profundidades de inundación y velocidades de la corriente, obtenidas mediante la modelación del tsunami de 1960, permitió espacializar la amenaza de tsunami diferenciada (ATD). Efectivamente, la peligrosidad de un tsunami al interior del área de inundación, se comporta de forma variada. Esta información permitió identificar los sectores donde un tsunami será más peligroso.

La espacialización de la exposición (E) ante tsunami permite reconocer los lugares más expuestos. Esta capa-objetivo integró los criterios localización en zona costera, distancia a sitio seguro, pendiente del terreno y altitud. Debido a las características particulares del área de estudio, la exposición a tsunamis en general es media a alta. La razón se explica principalmente por su carácter de estuario, de topografía plana y bajas altitudes. Donde las zonas de seguridad, con altitudes superiores a $10 \mathrm{~m}$, son escasas y distantes para gran parte de la población.

La vulnerabilidad socioeconómica (VS) ayuda a identificar quiénes son vulnerables en Maullín. Independiente de la magnitud de un tsunami, en territorios en riesgo, generalmente la población pobre y la numerosa concentración de habitantes se relaciona con altas probabilidades de daño (UN-EIRD, 2004).

La integración de la exposición (E) con la vulnerabilidad socioeconómica (VS) permitió espacializar la vulnerabilidad (V). La función de este modelo espacial fue identificar idónde se es más vulnerable?, matriz que combinada con la amenaza de tsunami diferenciada (ATD), nos genera la zonificación de riesgo de tsunami (ZRT).

La ZRT indica que el riesgo más alto en el curso inferior del río Maullín, se concentra principalmente en San Pedro Nolasco (Ver Figura $\mathrm{N}^{\circ} 6$ ), sector que presenta los mayores niveles de vulnerabilidad física, elemento que combinado con su localización, en un 
Figura $\mathrm{N}^{\circ} 5$

Factores de amenaza y vulnerabilidad

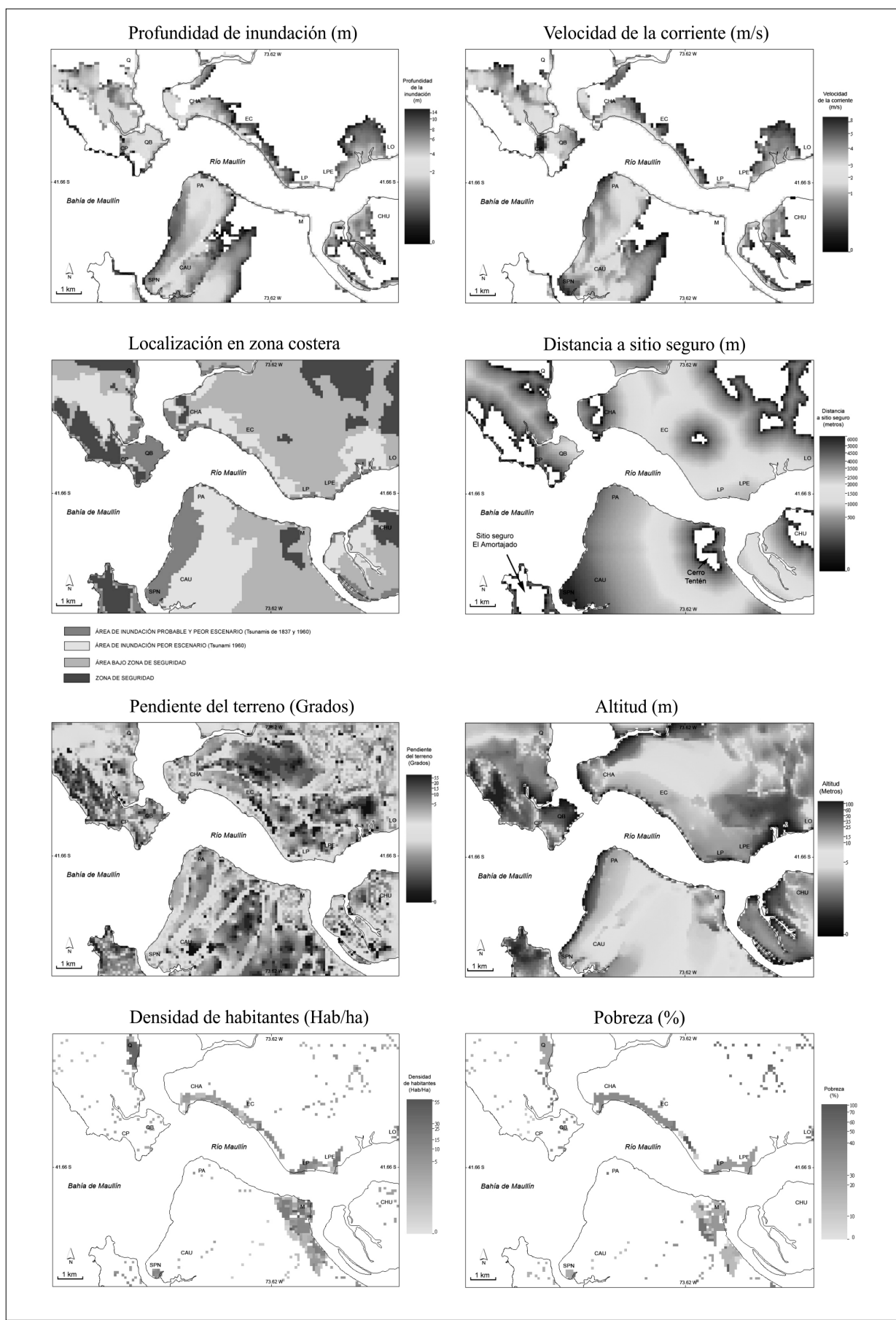

Sectores: Caleta Pichicuyen (CP), Quenuir (Q), Quenuir bajo (QB), San Pedro Nolasco (SPN), Caulle (CAU), Chanhué (CHA), Pangal (PA), El Carrizo (EC), La Pasada (LP), Maullín (M), Lepihué (LPE), Chuyaquén (CHU) y Lolcura (LO).

Fuente: Elaboración propia. 
Figura $\mathrm{N}^{\circ} 6$

Capas-objetivo y zonificación de riesgo de tsunami

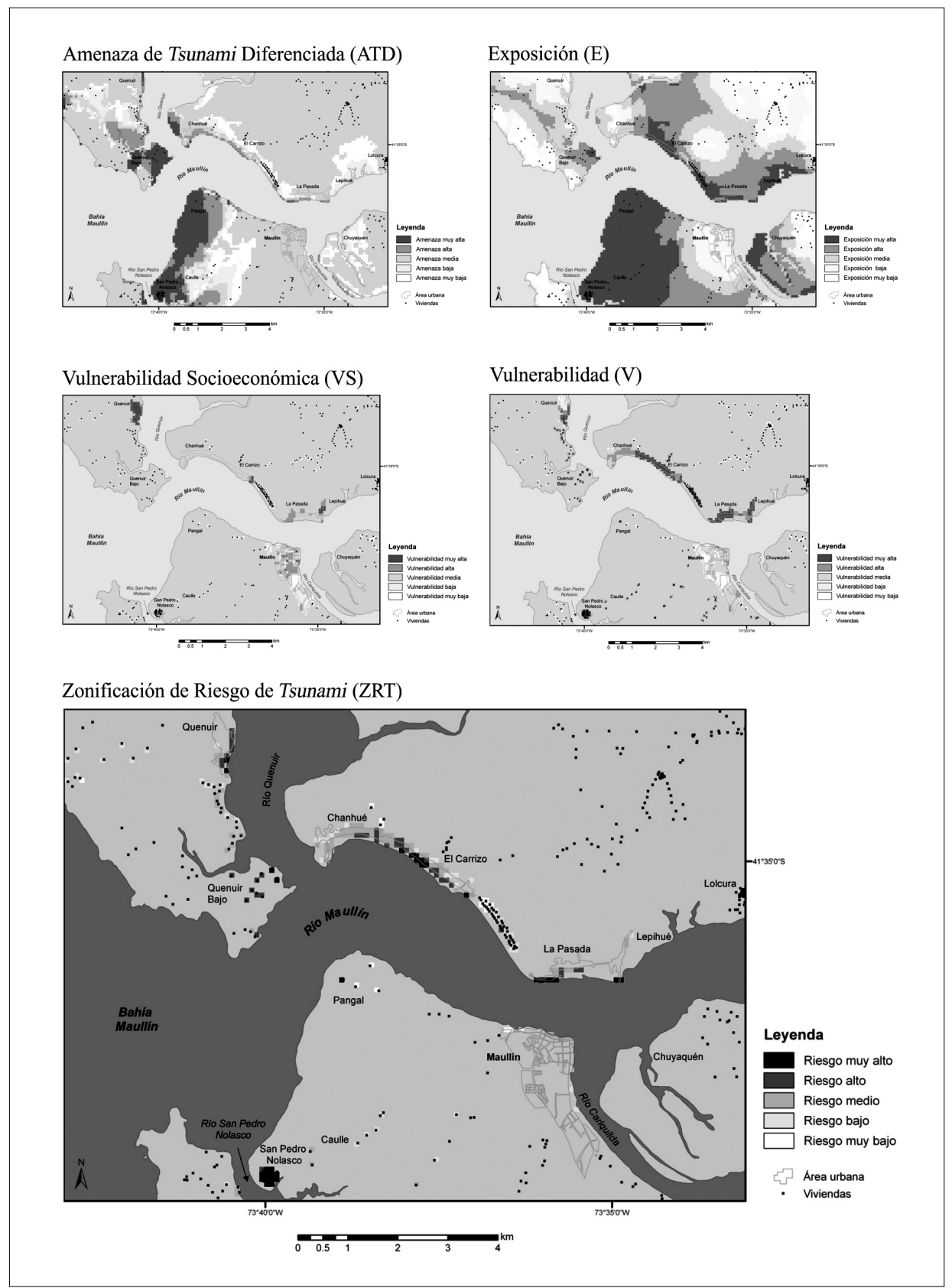

Fuente: Elaboración propia.

área de amenaza de tsunami diferenciada muy alta, permiten comprender su elevado riesgo. Otros sectores que también presentan un nivel muy alto de riesgo, se localizan en la ribera norte del río Maullín; involucrando sectores ribereños específicos de El Carrizo y La Pasada. Lo mismo ocurre con puntuales viviendas emplazadas en Quenuir bajo y asentamientos ribereños en Quenuir. 
Particularmente, la ribera norte del río Maullín, entre Chanhué y La Pasada, presenta un riesgo diferenciado. En el sector de Chanhué predomina el riesgo medio, aumentando en dirección hacia el sector El Carrizo. Luego, entre El Carrizo y La Pasada, el riesgo alcanza niveles bajos y muy bajos, para aumentar a riesgo muy alto en La Pasada. El sector de Lepihué no presenta riesgo debido a que actualmente no está habitado, sin embargo, se debe considerar que el tsunami de 1960 arrasó con las viviendas que se localizaban en ese sector. A diferencia, el poblado de Lolcura no presenta riesgo, debido a su localización en zonas ribereñas más elevadas. Efectivamente, Lolcura no fue afectado de forma importante por la inundación producida por el tsunami de 1960.

En general, el poblado de Maullín no presenta riesgo importante, principalmente debido a su emplazamiento, protegido de la acción directa de ondas de tsunami provenientes del océano Pacífico. En este contexto, su localización en la ribera sur del río Maullín, sumado a la escasa urbanización en zonas de baja altitud, se traduce en niveles de riesgo medio a muy bajo, afectando a específicos sectores ribereños del río Maullín y Cariquilda. Efectivamente, en el pueblo de MauIlín, el tsunami de 1960 solo inundó parte del camino a Pangal y el centro cívico. En tanto, también hubo una inundación parcial en las riberas del pueblo que dan al río Cariquilda.

Las viviendas aisladas que caracterizan el poblamiento del sector Caulle, presentan un riesgo medio en dirección al sector de San Pedro Nolasco, luego predomina el riesgo bajo. En Pangal, una vivienda presenta riesgo muy alto, rodeado de viviendas con riesgo muy bajo. Efectivamente, por su escaso poblamiento, en Pangal predomina el riesgo bajo, sin embargo, se debe considerar que en este sector funciona durante el verano el principal camping de Maullín, lugar que puede llegar a concentrar una población flotante de decenas de personas, hecho que incrementaría los niveles de riesgo.

\section{Conclusiones}

Comprender cómo se comportará la inundación de un potencial tsunami y cuáles serán sus probables consecuencias en una comunidad, particularmente hoy, se transforma en información de base para el ordenamiento de territorios costeros, el diseño de medidas de mitigación y el manejo de emergencias.

En la presente aplicación se ha integrando un conjunto de técnicas y criterios propuestos por la investigación científica de tsunamis, permitiendo zonificar un escenario de riesgo de tsunami para el curso inferior del estuario del río Maullín.

Las funciones propuestas para valorar criterios de amenaza y vulnerabilidad ante tsunami, son un paso adelante en el complejo estudio de la espacialización de multifactores que integran la comprensión del riesgo. Se destaca el uso del criterio pobreza, como un factor de vulnerabilidad que puede aproximarse a los múltiples agentes que confluyen en la vulnerabilidad socioeconómica.

Los resultados de la zonificación de riesgo de tsunami, evidencian cómo las características del lugar, su localización, el comportamiento diferenciado de la amenaza, y los mayores porcentajes de pobreza y su concentración condicionan los niveles de riesgo. Esta información se transforma en la base para futuros estudios de ordenamiento territorial que permitan disminuir los niveles de riesgo identificados en el área de estudio.

La combinación del conjunto de procedimientos utilizados para discriminar el riesgo de tsunami, se presenta como una metodología que puede ser aplicada en otras localidades costeras. Su estructura permite con claridad identificar los input compuestos de factores de amenaza y vulnerabilidad; su integración mediante técnicas de evaluación multicriterio; y su resultado correspondiente a la zonificación de riesgo de tsunami.

\section{Referencias bibliográficas}

ANDRADE, B.; ARENAS, F. y LAGOS, $M$. Incorporación de criterios de fragilidad ambiental y riesgo en la planificación territorial de la costa de Chile central. Revista de Geografía Norte Grande, 2010, № 45, p. 5-20. 
ARCAS, D.; CHAMBERLIN, C.; LAGOS, M.; RAMÍREZ, M.; TANG, L. \& WEI, Y. Near field modeling for the Maule Tsunami from DART, GPS and finite solutions, Abstract U21B-07. Fall Meeting AGU, 2010, San Francisco, California.

ATWATER, B.; CISTERNAS, M.; BOURGEOIS, J.; DUDLEY, W.; HENDLEY, J. \& STAUFFER, P. Surviving a Tsunami - Lessons from Chile, Hawaii, and Japan. Circular 1187 USGS, 1999, p. 18.

BARRIENTOS, S. \& WARD, S. The 1960 Chile earthquake: Inversion for slip distribution from surface deformation. Geophysical Journal International, 1990, 103, p. 589- 598.

BERNARD, E. Tsunami: Reduction Of Impacts through three Key Actions (TROIKA). Proceedings of the International Tsunami Symposium 2001 (ITS 2001), 2001, Session 1-1, Seattle, WA, p. 247-262.

BERNARD, E. \& ROBINSON, A. (Editors). The Sea: Tsunamis. Cambridge: Ed. Harvard University Press, 2009, Vol. 15, p. 443.

CHEN. K.; BLONG, R. \& JACOBSON, C. MCE-RISK: integrating multicriteria evaluation and GIS for risk decision-making in natural hazards. Environmental Modelling \& Software, 2001, Vol. 16, N 4, p. 387-397.

CIFUENTES, I. The 1960 Chilean earthquakes. Journal of Geophysical Research, 1989, Vol. 94, p. 665-680.

CISTERNAS, M.; ATWATER, B.; TORREJÓN, F.; SAWAI, Y.; MACHUCA, G.; LAGOS, M.; EIPERT, A.; YOULTON, C.; SALGADO, I.; KAMATAKI, T.; SHISHIKURA, M.; RAJENDRAN, CP.; MALIK, J.; RIZAL, Y. \& HUSNI, M. Predecessors of the giant 1960 Chile earthquake. Nature, 2005, Vol. 437, p. 404407.

DAO, M. \& TKALICH, P. Tsunami propagation modeling - a sensitivity study. Natural Hazards and Earth System Sciences, 2007, Vol.7, p. 741-754.

DENGLER, L.; LUDY, B. \& PATTON, J. Relative Tsunami Hazard Maps, Humboldt County, California. Eos Transactions, AGU,
2003, Vol. 84, N 46, Fall Meeting Supplement, Abstract OS22B-1162.

DEPARTAMENTO DE NAVEGACIÓN E HIDROGRAFÍA DE LA ARMADA DE CHILE (DNHA). El maremoto del 22 de mayo de 1960 en las costas de Chile. Valparaíso: Departamento de Navegación e Hidrografía de la Armada de Chile, 1961.

FRITZ, H.; PETROFF, C.; CATALÁN, P.; CIENFUEGOS, R.; WINCKLER, P.; KALLIGERIS, N.; WEISS, R.; BARRIENTOS, S.; MENESES, G.; VALDERAS-BERMEJO, C.; EBELIG, C.; PAPADOPOULOS, A.; CONTRERAS, M.; ALMAR, R.; DOMÍNGUEZ, J. \& SYNOLAKYS, C. Field Survey on the 27 February 2010 Chilean Tsunami. Pure and Applied Geophysics, 2011, No 168:1989-2010.

GHRIBI, M. GIS Applications for Monitoring Environmental Change and Supporting Decision-making in Developing Countries. Trieste: International Centre for Science and High Technology, United Nations Industrial Development Organization, 2005.

GONZÁLEZ, F.; TITOV, V.; MOFJELD, H.; VENTURATO, A.; SCOTT, R.; HANSEN, R.; COMBELLICK, R.; EISNER, R.; HOIRUP, D.; YANAGI, B.; YONG, S.; DARIENZO, M.; PRIEST, G.; CRAWFORD, G. \& WALSH, T. Progress in NTHMP Hazard Assessment. Natural Hazards, 2005, Vol. 35, N 1, p. 89-110.

GOTO, C.; OGAWA, Y.; SHUTO, N. \& IMAMURA, F. Numerical Method of Tsunami Simulation with the Leap-Frog Scheme. Paris: IUGG/IOC TIME Project Intergovernmental Oceanographic Commission of UNESCO, Manuals and Guides 35, 1997, Paris, 4 Parts.

INSTITUTO LATINOAMERICANO Y DEL CARIBE DE PLANIFICACIÓN ECONÓMICA Y SOCIAL (ILPES). La identificación de proyectos y bolsones de pobreza a nivel local. Guía para utilizar la base de datos del censo en REDATAM+. Santiago de Chile: Instituto Latinoamericano y del Caribe de Planificación Económica y Social (ILPES), Dirección de Proyectos y Programación de Inversiones, CEPAL/CELADE, 1995.

INSTITUTO NACIONAL DE ESTADÍSTICAS (INE). Base de datos Censo 2002. San- 
tiago de Chile: Instituto Nacional de Estadísticas, [archivo digital REDATAM], 2003.

IMAMURA, F. Tsunami modeling: calculating inundation and hazard maps. Chapter 10. In: BERNARD, E. \& ROBINSON, A. (Editors). The Sea: Tsunamis. Cambridge: Ed. Harvard University Press, 2009, Vol. 15, p. 321-332.

INTERGOVERNMENTAL OCEANOGRAPHIC COMMISSION (IOC). Tsunami preparedness-Information guide for disaster planners. UNESCO, Intergovernmental Oceanographic Commission, Manuals and Guides No49, 2008.

INTERGOVERNMENTAL OCEAN OGRAPHIC COMMISSION (IOC). HaZard Awareness and Risk Mitigation in Integrated Coastal Management (ICAM). Paris: UNESCO, Intergovernmental Oceanographic Commission, Manual and Guides $N^{\circ} 50$, 2009.

KANAMORI, H. \& CIPAR, J. Focal process of the great Chilean earthquake may 22, 1960. Physics of the Earth and Planetary Interiors, 1974, Vol. 9, p. 128-136.

KOSHIMURA, S. \& YANAGISAWA, H. Developing fragility functions for tsunami damage estimation using the numerical model and satellite imagery. 5th International Workshop on Remote Sensing Applications to Natural Hazards, Washington DC, 2007, p. 1-6.

LAGOS, M. y CISTERNAS, M. Depósitos de tsunami como indicadores de riesgo: evidencias sedimentarias. Revista Geográfica de Chile Terra Australis, 2004, № 49, p. 329351.

LAGOS, M.; CISTERNAS, M. y MARDONES, M. Construcción de viviendas sociales en áreas de riesgo de tsunami. Revista de la Construcción, 2008, Vol. 7, № 2, p. 4-16.

LAGOS, M. y CISTERNAS, M. EI nuevo riesgo de tsunami: considerando el peor escenario. Scripta Nova, Revista Electrónica de Geografía y Ciencias Sociales, 2008, Vol. XII, $N^{\circ} 270$ (29). Disponible en Internet: http:// geocritica.com/
LAGOS, M.; RAMÍREZ, M.; ARCAS, D.; GARCÍA, C. \& SEVERINO, R. Magnitude and Impact from the 2010 Chilean Tsunami. Eos Transactions, AGU, 2010, Vol. 91, No 26, Meet. Am. Suppl., Abstract U41A-18.

LAGOS, M.; ARENAS, F.; LILLO, I.; HENRÍQUEZ, C.; QUENSE, J. \& GARCÍA, C.; SEVERINO, R. Modelación de nuevos escenarios de peligro de tsunami para localidades del Centro-Sur de Chile. XXXII Congreso Nacional y XVII Internacional de Geografía, 2011, Valparaíso, Chile.

LAGOS, M. \& HARO, J. Magnitude and impact from the 2011 Tohoku Tsunami in the coast of Chile. Fall Meeting, AGU, 2011, Abstract $\mathrm{NH} 23-1209813$

PROGRAMA DE LAS NACIONES UNIDAS PARA EL DESARROLLO (PNUD). Desarrollo sin pobreza. II Conferencia Regional sobre la pobreza en América Latina y el Caribe, 1990, Quito, Ecuador.

SAATY, R. The analytic hierarchy process: what it is and how it is used. Mathematical Modeling, 1987, Vol. 9, №3, p. 161-176.

SATAKE. K.; OKAL, E. \& BORRERO, J. Tsunami and its hazard in the Indian and Pacific Oceans: introduction. Pure Applied Geophysics, 2007, DOI10.1007/s00024-006-0163-6.

SATO, H.; MURAKAMI, H.; KOZUKI, Y.; YAMAMOTO, N. Study on a Simplified Method of Tsunami Risk Assessment. Natural Hazards, 2003, Vol. 29 No 3, p. 325-340.

SERVICIO HIDROGRÁFICO Y OCEANOGRÁFICO DE LA ARMADA (SHOA). Tablas de Marea de la Costa de Chile. Valparaíso: Servicio Hidrográfico y Oceanográfico de la Armada, Publicaciones Náuticas 3009, 2003.

SHUTO, N. Tsunami intensity and disasters. TINTI, S. (Editor). Tsunamis in the World. Dordrecht: Kluwer Academic Publishers, 1993, p. 197-216.

SIEVERS, H.; VILLEGAS, G. \& BARROS, B. The seismic sea wave of 22 may 1960 along the Chilean coast. Bulletin of the Seismological Society of America, 1963, Vol. 53, p. 1125-1190. 
SINOLAKIS, C. \& KANOGLU, U. Tsunami modeling: development of benchmarked models. Chapter 8. In: BERNARD, E. \& ROBINSON, A. (Editors). The Sea: Tsunamis. Cambridge: Ed. Harvard University Press, 2009, Vol. 15, p. 237-294.

SUGAWARA, D. \& GOTO, K. Numerical modeling of the 2011 Tohoku-oki tsunami in the offshore and onshore of Sendai Plain, Japan. Sedimentary Geology, 2012. Disponible en Internet: http://dx.doi.org/10.1016/j.sedgeo.2012.08.002

SUGIMOTO, T.; MURAKAMI, H.; KOZUKI, Y.; NISHIKAWA, K. \& SHIMADA, T. A Human Damage Prediction Method for Tsunami Disasters Incorporating Evacuation Activities. Natural Hazards, 2003, Vol. 29, №3, p. 587 602.

PONTIFICIA UNIVERSIDAD CATÓLICA DE CHILE (UC). Definición de áreas de peligro de tsunami diferenciado para las localidades de Talcahuano, Llico, Tubul, Dichato y Constitución. Santiago de Chile: Ministerio de Vivienda y Urbanismo, Gobierno de Chile/ Instituto de Geografía, Laboratorio de Investigación de Tsunami, Pontificia Universidad Católica de Chile, 2010a.

PONTIFICIA UNIVERSIDAD CATÓLICA DE CHILE (UC). Estudio de riesgo de sismos y maremoto para comunas costeras de las Regiones de O'Higgins y del Maule. Santiago de Chile: Subsecretaria de Desarrollo Regional y Administrativo del Ministerio del Interior, Gobierno de Chile/Instituto de Estudios Urbanos y Territoriales y Observatorio de Ciudades UC, Facultad de Arquitectura, Diseño y Estudios Urbanos, Pontificia Universidad Católica de Chile, 2010b.
UNIVERSIDAD DEL BÍO-BÍO (UBB). Estudio de riesgos de sismos y maremoto para comunas Costeras de la Región del Biobío. Concepción: Subsecretaria de Desarrollo Regional y Administrativo del Ministerio del Interior, Gobierno de Chile / Departamento de Planificación y Diseño Urbano, Laboratorio de Estudios Urbanos, Facultad de Arquitectura, Construcción y Diseño, Universidad del Bío-Bío, 2010.

INTER-AGENCY SECRETARIAT OF THE INTERNATIONAL STRATEGY FOR DISASTER REDUCTION (UN-ISDR). Living with Risk, A global review of disaster reduction initiatives. Inter-Agency Secretariat of the International Strategy for Disaster Reduction, 2004, p. 382.

VENTURATO, A.; TITOV, V.; MOFJELD, $\mathrm{H}$. \& GONZALEZ, F. NOAA TIME Eastern Strait of Juan de Fuca, Washington, Mapping Project: Procedures, Data Sources, and Products. Contribution 2713 from NOAA/Pacific Marine Environmental Laboratory, 2004, p. 26.

WALSH, T.; TITOV, V.; VENTURATO, A.; MOFJELD, H. \& GONZÁLEZ, F. Tsunami Hazard Map of the Anacortes -Whidbey Island Area. Washington: Modeled Tsunami Inundation from a Cascadia Subduction Zone Earthquake. Washington Division of Geology and Earth Resources and NOAA TIME Center PMEL. Open File Report 2005-1, 2005, scale $1: 62.500$.

YAMAZAKI, Y.; CHEUNG, K. \& KOWALIK, Z. Depth-integrated, non-hydrostatic model with grid nesting for tsunami generation, propagation and run-up. International Journal for Numerical Methos in Fluids, 2010, DOI:10.1002/fld.2485. 
